\title{
Multimodal Voxel-Based Meta-Analysis of White Matter Abnormalities in Obsessive-Compulsive Disorder
}

\author{
Joaquim Radua*, , , Mar Grau ${ }^{1,3}$, Odile A van den Heuvel $^{4}$, Michel Thiebaut de Schotten ${ }^{5,6}$, Dan J Stein ${ }^{7}$, \\ Erick J Canales-Rodríguez ${ }^{2}$, Marco Catani ${ }^{5}$ and David Mataix-Cols ${ }^{1,8}$ \\ 'Department of Psychosis Studies, Institute of Psychiatry, King's College London, London, UK; ${ }^{2}$ Research Unit, FIDMAG Germanes \\ Hospitalàries - CIBERSAM, Sant Boi de Llobregat, Barcelona, Spain; ${ }^{3}$ Westminster and Kensington and Chelsea Early Intervention in Psychosis \\ Team, Central North West London NHS Trust, London, UK; ${ }^{4}$ Department of Psychiatry and Department of Anatomy and Neurosciences, VU \\ University Medical Center, Amsterdam, The Netherlands; ${ }^{5}$ Natbrainlab_-Department of Forensic and Neurodevelopmental Sciences, Institute of \\ Psychiatry, King's College London, London, UK; ' UMR_S 975-CNRS UMR 7225, Centre de Recherche de l'Institut du Cerveau et de la Moelle \\ épinière, Groupe Hospitalier Pitié-Salpêtrière, Paris, France; ' Department of Psychiatry and Mental Health, Faculty of Health Sciences, University \\ of Cape Town, Cape Town, South Africa; ${ }^{8}$ Department of Clinical Neuroscience, Karolinska Institutet, Stockholm, Sweden
}

\begin{abstract}
White matter (WM) abnormalities have long been suspected in obsessive-compulsive disorder (OCD) but the available evidence has been inconsistent. We conducted the first multimodal meta-analysis of WM volume (WMV) and fractional anisotropy (FA) studies in OCD. All voxel-wise studies comparing WMV or FA between patients with OCD and healthy controls in the PubMed, ScienceDirect, Google Scholar, Web of Knowledge and Scopus databases were retrieved. Manual searches were also conducted and authors were contacted soliciting additional data. Thirty-four data sets were identified, of which 22 met inclusion criteria (five of them unpublished; comprising 537 adult and pediatric patients with $O C D$ and 575 matched healthy controls). Whenever possible, raw statistical parametric maps were also obtained from the authors. Peak and raw WMV and FA data were combined using novel multimodal meta-analytic methods implemented in effect-size signed differential mapping. Patients with OCD showed widespread WM abnormalities, but findings were particularly robust in the anterior midline tracts (crossing between anterior parts of cingulum bundle and body of corpus callosum), which showed both increased WMV and decreased FA, possibly suggesting an increase of fiber crossing in these regions. This finding was also observed when the analysis was limited to adult participants, and especially pronounced in samples with a higher proportion of medicated patients. Therefore, patients with OCD may have widespread WM abnormalities, particularly evident in anterior midline tracts, although these changes might be, at least in part, attributable to the effects of therapeutic drugs.

Neuropsychopharmacology (2014) 39, I547-1557; doi:I0.1038/npp.20I4.5; published online 5 February 20I4
\end{abstract}

Keywords: cingulum bundle; diffusion tensor imaging; multimodal meta-analysis; obsessive-compulsive disorder; superior longitudinal fasciculus; voxel-based morphometry

\section{INTRODUCTION}

Obsessive-compulsive disorder (OCD) is a chronic and relatively prevalent neuropsychiatric disorder, which causes much distress and disability. Although the precise causes of the disorder remain unknown, a large literature has emphasized the importance of specific fronto-striatal neurocircuitry in mediating its symptoms, including early reports of obsessive-compulsive symptoms developing after brain lesions, studies on neuropsychological deficits in individuals with OCD, as well as a range of functional neuroimaging studies exposing patients to illness-relevant cues

\footnotetext{
*Correspondence: Dr J Radua, Department of Psychosis Studies, Institute of Psychiatry, King's College London, PO 69, 16 De Crespigny Park, London SE5 8AF, UK, Tel: +44 (0) 207848 0363, Fax: +44 (0) 207848 0379, E-mail: joaquim.radua@kcl.ac.uk Received I4 September 20 13; revised 30 December 2013; accepted 2 January 2014; accepted article preview online 10 January 2014
}

(Daniele et al, 1997; Mataix-Cols and van den Heuvel, 2006; Saxena and Rauch, 2000).

The findings of structural neuroimaging studies have been broadly consistent with the prevailing fronto-striatal model of OCD. For example, various meta-analyses of voxel-based morphometry (VBM) studies consistently found decreased gray matter volumes in dorsal mediofrontal/anterior cingulate cortex, and increased gray matter volumes in basal ganglia and parietal cortex (Radua and Mataix-Cols, 2009; Radua et al, 2010; Rotge et al, 2009). A recent multicenter mega-analysis found robust decreases of gray matter volume in dorsomedial prefrontal/anterior cingulate cortex and inferior frontal gyrus extending to anterior insula, age-related volume decreases in the temporal cortex extending to thalamus, volume increases in the cerebellum, and age-related volume increases in the basal ganglia (de Wit et al, 2013). Recent reviews have also highlighted the importance of other brain structures such as 
parietal and limbic areas in the neuroanatomy of the disorder (Menzies et al, 2008a; Milad and Rauch, 2011). Finally, a growing literature additionally highlights the importance of considering entire brain systems and the inter-connectivity between brain regions, rather than discrete brain regions in OCD (Harrison et al, 2009; Menzies et al, 2008a).

Although research on gray matter abnormalities in OCD has seen considerable progress, the study of white matter (WM) tracts that connect the brain regions implicated in the disorder have received considerably less attention (Fontenelle et al, 2009). A careful characterization of WM abnormalities in OCD is important to help gaining a deeper understanding of the brain systems implicated in the disorder. Some of the published VBM studies included information on WM volumes (WMVs), with results that are not easy to reconcile at first glance (Duran et al, 2009; Lazaro et al, 2011; van den Heuvel et al, 2009). In the abovementioned mega-analysis, de Wit et al (2013) found between-group differences in frontal WMV, presumably reflecting abnormalities in WM tracts connecting prefrontal and subcortical brain regions, thus being broadly consistent with the prevailing fronto-striatal model of OCD, and in line with some, but not all, previous WMV studies (Togao et al, 2010; van den Heuvel et al, 2009). To our knowledge, no meta-analyses of WMV studies have been conducted to date.

Diffusion tensor imaging (DTI) is potentially more suited to study the integrity of WM tracts in OCD. A growing number of such studies have been published, although these studies have been typically small, their methods diverse and their results inconsistent. Some of these inconsistencies may simply be related to the study of pediatric and adult samples, as important changes in WM occur during normal brain development (Peters et al, 2012); therefore pediatric and adult studies may not be directly comparable. Another potentially important confound is whether patients are on medication at the time of their scan. At least two small studies (Fan et al, 2012; Yoo et al, 2007) found that WM alterations in OCD may be partially reversible with pharmacotherapy, although these studies lacked a control (placebo) group and therefore these changes could not be unequivocally linked to the treatment itself. In an attempt to summarize the growing yet confusing DTI literature, a recent meta-analysis of seven DTI studies in OCD (Peng et al, 2012) found lower fractional anisotropy (FA) in the cingulum bundle, the inferior fronto-occipital fasciculus and the superior longitudinal fasciculus (SLF), and increased FA in the left uncinate fasciculus. Given the modest number of included studies, these findings should be regarded as preliminary.

The current meta-analysis aimed to provide an updated quantitative summary of studies investigating WMV and FA abnormalities in OCD using effect-size signed differential mapping (ES-SDM), a meta-analytic technique capable of combining studies in which only peak information is available, with studies from which the raw statistical parametric maps (SPMs) can be obtained from the authors (Radua et al, 2012b). We also used novel multimodal meta-analytical methods, which enable the combination of various imaging modalities in the same meta-analysis, thus potentially offering insights that are not apparent from any given imaging modality alone (Radua et al, 2013b). Specifically, we first conducted separate meta-analyses of WMV and FA studies in OCD, followed by a multimodal meta-analysis of WMV and FA. Finally, we used meta-regression methods to examine the potentially moderating effects of medication and other relevant variables on the reported abnormalities.

\section{MATERIALS AND METHODS}

\section{Inclusion of Studies}

A comprehensive literature search of WM VBM and diffusion-weighted magnetic resonance imaging studies in OCD was conducted using PubMed, ScienceDirect, Google Scholar, Web of Knowledge and Scopus search engines. The search keywords were 'obsessive-compulsive disorder' plus 'voxel-based morphometry', 'white matter', 'volume', 'diffusion tensor', 'DTI' or 'anisotropy'. In addition, manual searches were conducted within review papers and reference sections of individual papers. All voxel-wise studies comparing WMV or FA between individuals with OCD and healthy controls were eligible for inclusion. Exclusion criteria were: (1) studies from which peak coordinates or parametric maps could not be retrieved from the published article or after contacting the authors; (2) studies limiting their analyses to specific regions of interest (ROI); (3) studies in which different thresholds were used in different regions of the brain; and (4) studies using tract-based spatial statistics (TBSS). The latter criterion was required because TBSS studies restrict their analyses to a FA-derived tract skeleton and thus could not be combined with (whole-brain) WMV and FA studies.

As WM substantially changes during development, especially in the SLF, but also in the inferior longitudinal and fronto-occipital fasciculi and the anterior thalamic radiation (Peters et al, 2012), the meta-analyses were also conducted limiting the analyses to adult studies (ie, after the exclusion of pediatric/adolescent samples).

\section{Data Extraction}

Data were independently extracted from each study by one of the investigators (MG) and checked by another investigator (JR) to minimize data entry errors. Corresponding authors were asked whether they could provide additional details not included in the original publications. MOOSE guidelines for meta-analyses of observational studies in epidemiology were followed in the study.

\section{Global WMV}

Differences in global WMV between patients and controls were meta-analyzed using standard restricted maximumlikelihood random-effects models as included in ES-SDM software (http://www.sdmproject.com/).

\section{Regional WMV}

Differences in regional WMV were also meta-analyzed using ES-SDM software (Radua et al, 2011), which has specific WMV and FA templates (Radua et al, 2011). Voxel-based meta-analytic methods have been described in detail 
elsewhere (Radua et al, 2012b) and thus only the main features of ES-SDM are summarized here.

First, as stated in the exclusion criteria, only studies in which the same threshold is used throughout the whole brain are included, in order to avoid biases toward those brain regions that authors may have liberally thresholded (Radua and Mataix-Cols, 2009). Second, meta-analytic WM maps are based on a specific WM template, rather than on whole brain or gray matter templates, which would bias the statistical significance (Radua et al, 2011). Third, both increases and decreases of WMV are recreated in the same map to avoid WMV from regions with high between-study heterogeneity appearing as increased and decreased at the same time. Fourth, meta-analytical calculations are based on random-effects models, thus taking sample size, intrastudy variability and between-study heterogeneity into account (Radua et al, 2012b). Fifth, assessment of statistical significance is based on a distribution-free permutation test (Radua et al, 2012b). Finally, results are thresholded at $P<0.005$, requiring a peak $Z>1$ and a cluster extent of 10 voxels; this particular set of thresholds was found to optimize sensitivity while correctly controlling false-positive rate in the empirical validation of ES-SDM (Radua et al, 2012b).

The main analyses were complemented with analyses of robustness, namely: (a) jackknife sensitivity analysis, consisting of iteratively repeating the meta-analysis excluding one study at a time, in order to establish whether the results are replicable (Radua and Mataix-Cols, 2009); (b) repetition of the meta-analysis after the exclusion of pediatric/ adolescent samples; and (c) funnel plots to detect gross abnormalities and assess whether findings might have been driven by few or small studies.

\section{Regional FA}

The meta-analysis of regional FA was methodologically identical to that of regional WMV. However, the main analyses were also complemented with meta-regressions by the mean OCD symptom severity (as measured by the YaleBrown obsessive-compulsive scale (YBOCS)), the mean age of OCD individuals, and the percentage of OCD individuals receiving medication. The equivalent analyses could not be conducted in regional WMV because of an insufficient number of studies. As in previous studies (Radua et al, 2012a), results from meta-regressions were thresholded more conservatively $(P<0.0005)$ and only considered when significant slopes were accompanied by significant differences at one extreme.

\section{Multimodal Meta-Analysis}

Finally, the meta-analyses of regional WMV and FA abnormalities were combined in order to detect those brain regions showing differences in both imaging modalities. We followed the approach described in Radua et al (2013b), which aims to obtain the overlap between the abnormal regions in the two modalities accounting for noise in the meta-analytic $P$-values. Note that the aim of such analysis was not to detect correlations between WMV and FA, but rather, to detect those regions showing abnormalities in both WMV and FA. This method has already been successfully applied to the study of brain abnormalities in other psychiatric populations (Radua et al, 2012a).

\section{WM Structure Identification}

In order to identify the WM structures involved in the different analyses, meta-analytic results were projected into a high-resolution diffusion-weighted imaging data set using Advanced Normalisation Tools (ANTS, http://www.picsl. upenn.edu/ANTS/), which combine affine with diffeomorphic deformations (Avants et al, 2008). Further details on the acquisition and the processing of the high-resolution diffusion-weighted imaging data set can be found in Thiebaut de Schotten et al (2012) and Catani et al (2012). Meta-analytic results were also used to filter the whole-brain tractography of the diffusion-weighted imaging data set in order to display solely the WM structures of interest in the figures of this paper.

\section{RESULTS}

\section{Included Studies and Sample Characteristics}

As seen in Supplementary Figure 1, the search and contact with authors retrieved a total of 34 potentially suitable studies (13 WMV and 21 DTI). Of those, two WMV and four DTI studies were excluded because they used a ROI approach, six other DTI studies were excluded because they used TBSS, and one WMV study was excluded because its sample overlapped with the sample from another larger study. Please see Supplementary Figure 1 for references of the excluded articles.

Thus, 21 studies (providing 22 data sets: $10 \mathrm{WMV}$ and 12 DTI) completed before 31 January 2013 could be included in the meta-analysis (Table 1; Admon et al, 2012; Cannistraro et al, 2007; Carmona et al, 2007; Duran et al, 2009; Fan et al, 2012; Garibotto et al, 2010; Gruner et al, 2012; Ha et al, 2009; Koprivova et al, 2009; Lazaro et al, 2011; Li et al, 2011; Matsumoto et al, 2010; Menzies et al, 2008b; Nakamae et al, 2008; Pujol et al, 2004; Riffkin et al, 2005; Szeszko et al, 2005; Togao et al, 2010; van den Heuvel et al, 2009; Yoo et al, 2007, 2008). They comprised a total of 537 participants with OCD (469 adults and 68 children/adolescents) and 575 healthy controls (507 adults and 68 children/adolescents). No relevant differences between patients and healthy controls were found in terms of age and gender, as the original studies were already well matched in this respect. Raw SPMs could be retrieved for two studies (Nakamae et al, 2008; Togao et al, 2010), non-published peak data from three studies (Cannistraro et al, 2007; Lazaro et al, 2011; Yoo et al, 2008), and standard published peak data from the remaining studies. All the studies were of acceptable methodological quality (see Supplementary Tables 1-4) and written in English.

\section{Differences in Global WMV}

Data for this analysis were obtained from seven WMV data sets including 278 patients with OCD and 293 healthy controls (Koprivova et al, 2009; Lazaro et al, 2011; Matsumoto et al, 2010; Pujol et al, 2004; Togao et al, 2010; van den Heuvel et al, 2009; Yoo et al, 2008). This meta-analysis did not find differences in global WMV between patients and 
Table I Demographic Characteristics of the 22 Data Sets Included in the Meta-Analysis

\begin{tabular}{|c|c|c|c|c|c|c|c|c|c|c|c|c|c|c|c|}
\hline & \multicolumn{10}{|c|}{ Patients with OCD } & \multicolumn{5}{|c|}{ Comparison controls } \\
\hline & $\mathbf{N}$ & $\begin{array}{c}\text { Age } \\
\text { (mean) }\end{array}$ & Males & $\begin{array}{c}\text { Right } \\
\text { handed }\end{array}$ & $\begin{array}{c}\text { Years of } \\
\text { education }\end{array}$ & $\begin{array}{c}\text { Age of } \\
\text { onset }\end{array}$ & $\begin{array}{c}\text { Duration of } \\
\text { illness }\end{array}$ & $\begin{array}{l}\text { YBOCS } \\
\text { (mean) }\end{array}$ & $\begin{array}{l}\text { Drug free } \\
\text { (\%) }\end{array}$ & $\begin{array}{l}\text { Drug naive } \\
\text { (\%) }\end{array}$ & $\mathbf{N}$ & $\begin{array}{c}\text { Age } \\
\text { (mean) }\end{array}$ & Males & $\begin{array}{c}\text { Right } \\
\text { handed }\end{array}$ & $\begin{array}{c}\text { Years of } \\
\text { education }\end{array}$ \\
\hline \multicolumn{16}{|l|}{ White matter volume } \\
\hline Carmona et al (2007) & 18 & 12.9 & $72 \%$ & $83 \%$ & - & - & - & 21.4 & $44 \%$ & $44 \%$ & 18 & 13.0 & $72 \%$ & $83 \%$ & - \\
\hline Duran et al (2009) & 19 & 32.7 & $53 \%$ & $89 \%$ & - & 14.4 & 18.3 & 24.6 & $42 \%$ & $16 \%$ & 15 & 32.3 & $47 \%$ & $73 \%$ & - \\
\hline Koprivova et al (2009) & 14 & 28.6 & $36 \%$ & $100 \%$ & - & 13.9 & 15.6 & 16.4 & $29 \%$ & - & 15 & 28.7 & $40 \%$ & $100 \%$ & - \\
\hline Lazaro et al (201 I) & 27 & 15.6 & $56 \%$ & $100 \%$ & 9.8 & 13.0 & 2.4 & 16.7 & $0 \%$ & $0 \%$ & 27 & 16.1 & $48 \%$ & $100 \%$ & 10.3 \\
\hline Matsumoto et al (2010) & 16 & 32.8 & $44 \%$ & $88 \%$ & - & - & - & 22.0 & $63 \%$ & $25 \%$ & 32 & 32.6 & $44 \%$ & $84 \%$ & - \\
\hline Pujol et al (2004) & 72 & 29.8 & $56 \%$ & $85 \%$ & 13.2 & 17.0 & 13.0 & 26.7 & $25 \%$ & $7 \%$ & 72 & 30.1 & $56 \%$ & $85 \%$ & 14.0 \\
\hline Riffkin et al (2005) & 18 & 36.1 & $44 \%$ & $94 \%$ & 12.1 & - & - & 23.3 & $78 \%$ & - & 18 & 34.5 & $44 \%$ & $94 \%$ & 13.4 \\
\hline Togao et al (20|0) & 23 & 32.6 & $39 \%$ & $91 \%$ & 14.0 & 19.3 & 12.9 & 29.8 & $100 \%$ & $22 \%$ & 26 & 31.3 & $46 \%$ & $92 \%$ & 14.9 \\
\hline van den Heuvel et al (2009) & 55 & 33.7 & $29 \%$ & $89 \%$ & 15.3 & 14.4 & 18.7 & 22.8 & $100 \%$ & $44 \%$ & 50 & 31.4 & $40 \%$ & $90 \%$ & 16.2 \\
\hline Yoo et al (2008) & 71 & 26.6 & $66 \%$ & $96 \%$ & 14.0 & 18.6 & 8.0 & 22.8 & $17 \%$ & $17 \%$ & 71 & 26.7 & $66 \%$ & $100 \%$ & 15.2 \\
\hline \multicolumn{16}{|l|}{ Fractional anisotropy } \\
\hline Admon et al (2012) & 13 & 25.5 & $77 \%$ & $100 \%$ & 13.5 & - & 8 & 24.5 & $0 \%$ & $0 \%$ & 13 & 27.0 & $77 \%$ & $100 \%$ & 14.0 \\
\hline Cannistraro et al (200I) & 8 & 26.4 & $38 \%$ & $100 \%$ & 15.6 & - & - & 22.9 & $100 \%$ & - & 10 & 23.3 & $40 \%$ & $100 \%$ & 15.9 \\
\hline Fan et al (2012) & 27 & 25.5 & $63 \%$ & $100 \%$ & 14.0 & 20.4 & 4.8 & 22.0 & $100 \%$ & $48 \%$ & 23 & 28.8 & $65 \%$ & $100 \%$ & 14.6 \\
\hline Garibotto et al (2010) & 15 & 31.9 & $100 \%$ & $100 \%$ & 13.9 & 20.7 & 10.13 & 28.2 & $13 \%$ & - & 16 & 29.7 & $100 \%$ & $100 \%$ & 17.6 \\
\hline Gruner et al (2010) & 23 & 14.3 & $57 \%$ & $83 \%$ & - & - & - & 26.9 & $48 \%$ & $39 \%$ & 23 & 14.2 & $52 \%$ & $74 \%$ & - \\
\hline Ha et al (2009) check & 12 & 22.3 & $100 \%$ & - & - & - & - & 22.5 & - & - & 25 & 23.7 & $100 \%$ & - & - \\
\hline Ha et al (2009) clean & 10 & 24.5 & $100 \%$ & - & - & - & - & 20.2 & - & - & 25 & 23.7 & $100 \%$ & - & - \\
\hline Li et al (20I I) & 23 & 27.2 & $70 \%$ & $100 \%$ & 13.3 & 20.9 & 6.3 & 23.2 & $43 \%$ & $43 \%$ & 23 & 26.7 & $70 \%$ & $100 \%$ & 13.4 \\
\hline Menzies et al (2008) & 30 & 32.2 & $30 \%$ & $87 \%$ & - & - & - & 22.1 & $23 \%$ & - & 30 & 33.7 & $33 \%$ & $77 \%$ & - \\
\hline Nakamae et al (2008) & 15 & 29.7 & $60 \%$ & $93 \%$ & 15.2 & 19.6 & - & 29 & $0 \%$ & $0 \%$ & 15 & 29.1 & $60 \%$ & $93 \%$ & 15.7 \\
\hline Szeszko et al (2005) & 15 & 38.5 & $67 \%$ & $60 \%$ & 14.8 & 16.9 & 21.6 & 25.9 & $20 \%$ & - & 15 & 38.5 & $67 \%$ & $60 \%$ & 15.9 \\
\hline Yoo et al (2007) & 13 & 27.8 & $62 \%$ & $100 \%$ & 15.3 & 20.6 & 7.2 & 30.2 & $100 \%$ & $92 \%$ & 13 & 26.9 & $62 \%$ & $100 \%$ & 15.1 \\
\hline
\end{tabular}

Check and clean indicate the subsample of patients in this study. 
healthy controls $(\mathrm{d}=-0.07, Z=-0.88, P=0.379)$. Residual heterogeneity was neither significant $(\tau=0, Q=6.4$, $\mathrm{df}=6, P=0.381)$.

\section{Differences in Regional WMV}

Data for this analysis were obtained from all $10 \mathrm{WMV}$ data sets including 333 patients with OCD and 344 healthy controls (Carmona et al, 2007; Duran et al, 2009; Koprivova et al, 2009; Lazaro et al, 2011; Matsumoto et al, 2010; Pujol et al, 2004; Riffkin et al, 2005; Togao et al, 2010; van den Heuvel et al, 2009; Yoo et al, 2008). Patients showed significantly increased WMV in anterior midline WM structures, approximately corresponding to the corpus callosum (CC) and the cingulum bundle, as well as the right anterior thalamic radiation and the left arcuate fasciculus. Decreases of WMV were mainly observed around the right SLF, cortico-spinal tract, frontal aslant tract and anterior body of the CC. These findings were moderately robust in the jackknife analysis, in which they failed to emerge in 1 of the 10 combinations of 9 studies-findings were not statistically significant in combinations not including the study by Yoo et al (2008), one of the largest studies in the meta-analysis. The analysis after exclusion of pediatric/adolescent samples replicated most of the increases in anterior midline WM structures and right anterior thalamic radiation, but not the spatially largest decreases in right SLF, cortico-spinal tract, frontal aslant tract and anterior body of the CC. Table 2 provides a complete list of the findings and their details.

\section{Differences in Regional FA}

Data for this analysis were obtained from all 12 FA data sets including 204 patients with OCD and 231 healthy controls (Admon et al, 2012; Cannistraro et al, 2007; Fan et al, 2012; Garibotto et al, 2010; Gruner et al, 2012; Ha et al, 2009; Li et al, 2011; Menzies et al, 2008b; Nakamae et al, 2008; Szeszko et al, 2005; Yoo et al, 2007). Patients showed significantly decreased FA in midline WM structures such as the CC and the cingulum bundle, as well as parts of the right ventral SLF (SLF III) and a region also comprising left inferior longitudinal and fronto-occipital fasciculi. FA decreases in cingulum bundle and left inferior longitudinal and/or fronto-occipital fasciculi were spatially large $(>200$ voxels) and statistically robust, as they remained significant across all combinations of studies in the jack-knife analysis and after exclusion of pediatric/adolescent samples. We also found significantly increased FA in a region comprising parts of the SLF, cortico-spinal tract, frontal aslant tract and anterior body of the CC, a finding that was also observed after exclusion of pediatric/adolescents samples but failed to emerge in one of the combinations of studies in the jackknife analysis-it was not statistically significant after exclusion of the study by Menzies et al (2008b), one of the largest studies in the meta-analysis. Please see Table 2 for a complete list of the findings along with their details.

\section{Multimodal Analysis}

As shown in Table 2 and Figure 1, individuals with OCD showed: (a) increased WMV and FA in the parallel right thalamic radiation and cortico-spinal tract through the internal capsule; (b) increased WMV but decreased FA in midline WM structures, including the crossing between the anterior body of CC and cingulum bundle, as well as in regions comprising middle longitudinal fasciculus, inferior fronto-occipital fasciculus, forceps major of the CC, arcuate fasciculus and SLF; (c) decreased WMV but increased FA in the crossing of right SLF, cortico-spinal tract, frontal aslant tract and anterior body of the CC; and (d) decreased WMV and FA in right SLF III.

\section{Assessment of Publication Bias and Heterogeneity}

There was no significant between-study heterogeneity in any of the peaks of WM multimodal abnormality (largest $Z=0.92$ ). Visual inspection of their funnel plots did not reveal publication bias (Supplementary Figure 2), with no Egger test being statistically significant (lowest $P=0.085$ ). However, funnel plots showed that increases of WMV in right internal capsule and left middle longitudinal fasciculus were driven by only one study (Togao et al, 2010 and Yoo et al, 2008 respectively), and that FA abnormalities were generally driven by more studies than WMV abnormalities.

\section{Meta-Regressions}

Samples with higher percentages of medicated patients had more decreased FA in approximately the anterior midline region, which had been robustly detected as abnormal in the main analyses (slope peak at $-14,12,38, Z=-2.1, P=$ 0.00008 ; Figure 1). The blob comprised parts of the dorsal branch of the SLF (SLF I), the cortico-spinal tract, the CC and the local frontal connectivity (eg, U-shaped fibers). The meta-regression slope seemed to be especially driven by four relatively small studies with high percentages of medicated patients (Admon et al, 2012; Garibotto et al, 2010; Nakamae et al, 2008; Szeszko et al, 2005). As far as we could tell from the original publications, the processing pipeline in these four studies was of comparable quality to the remaining studies (mean $\mathrm{TE}=87 v s 80$, mean number of directions $=20$ vs 18, etc; for further details, see Supplementary Tables 3 and 4). The percentage of medicated patients was not associated with any other recorded demographic or clinical variable (eg, age, sex, etc) and was similar between pediatric and adult samples (52\% vs 56\%).

In addition, we also made the following observations: samples with younger patients had increased FA in the cingulum bundle, samples with older patients increased FA in the right dorsal prefrontal WM, and samples with higher mean YBOCS increased FA in the bilateral SLF. However, these results need to be interpreted cautiously because they were either driven by only few studies or did not clearly overlap with the between-group differences found in the main analyses.

\section{DISCUSSION}

The main finding of this meta-analysis was that patients with OCD appear to have widespread WM abnormalities, comprising parts of the cingulum bundle, CC, SLF, middle and inferior longitudinal fasciculi, anterior thalamic radiation, internal capsule, frontal aslant tract and/or inferior 
Table 2 Regions of Significant Differences in WMV and FA Between Patients with Obsessive-Compulsive Disorder and Healthy Controls

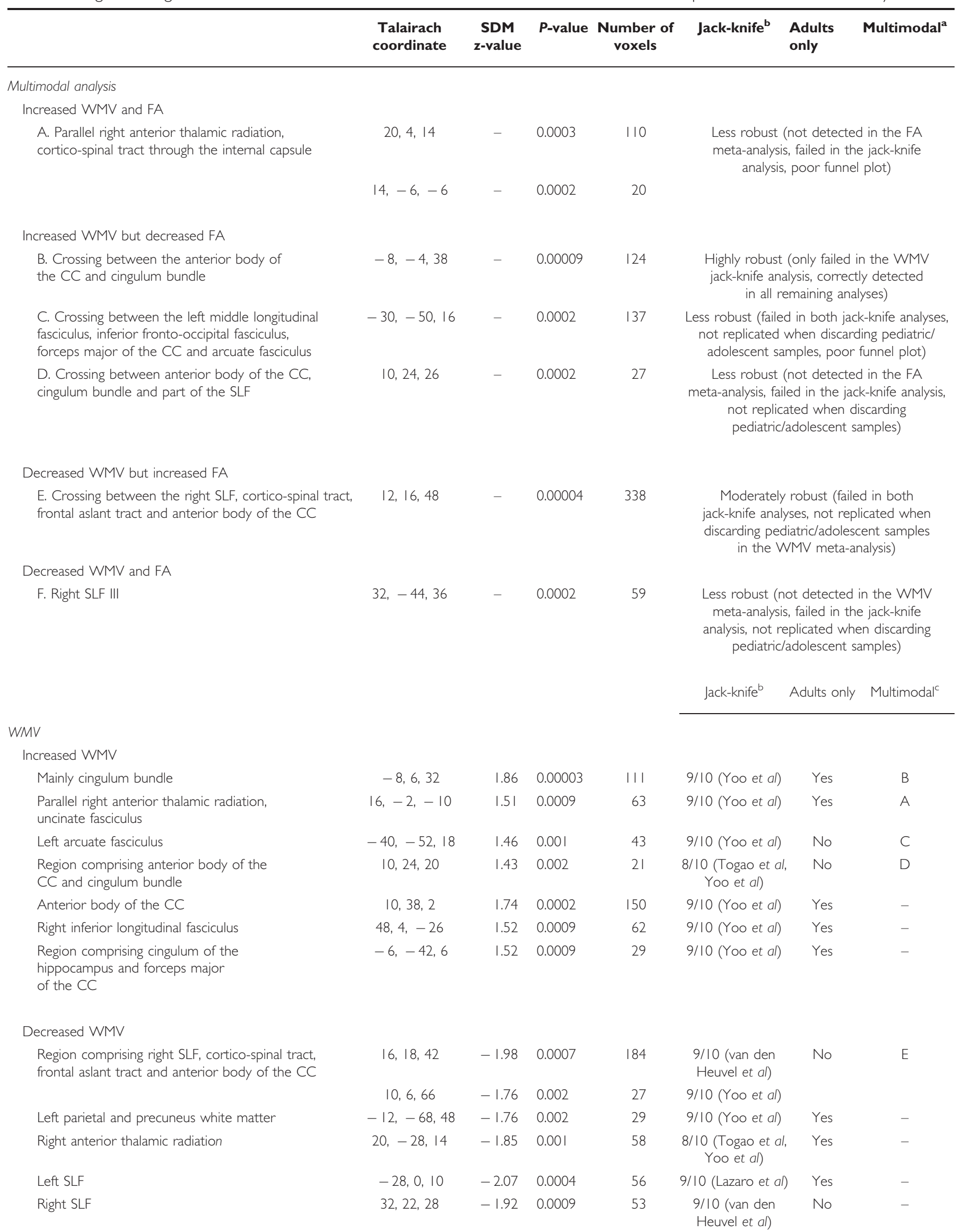


Table 2 (Continued)

\begin{tabular}{|c|c|c|c|c|c|c|c|}
\hline & $\begin{array}{l}\text { Talairach } \\
\text { coordinate }\end{array}$ & $\begin{array}{c}\text { SDM } \\
\text { z-value }\end{array}$ & P-value & $\begin{array}{c}\text { Number of } \\
\text { voxels }\end{array}$ & Jack-knife ${ }^{b}$ & $\begin{array}{l}\text { Adults } \\
\text { only }\end{array}$ & Multimodal $^{\mathrm{a}}$ \\
\hline \multicolumn{8}{|l|}{$F A$} \\
\hline \multicolumn{8}{|l|}{ Increased FA } \\
\hline $\begin{array}{l}\text { Region comprising right SLF, cortico-spinal } \\
\text { tract, frontal aslant tract and anterior body } \\
\text { of the CC }\end{array}$ & $26,6,46$ & 1.57 & 0.0007 & 47 & $\begin{array}{c}\text { 11/12 (Menzies } \\
\text { et al) }\end{array}$ & Yes & $E$ \\
\hline Mainly cingulum bundle & $-14,2,46$ & -2.02 & 0.00005 & 329 & $12 / 12$ & Yes & B \\
\hline Forceps major of the CC & $-26,-52,18$ & -1.65 & 0.0005 & 98 & $1 \mathrm{1} / 12$ (Fan et al) & No & C \\
\hline Right SLF III & $32,-48,34$ & -1.66 & 0.0005 & 68 & $\begin{array}{l}\text { I I/I2 (Szeszko } \\
\text { et al) }\end{array}$ & No & $\mathrm{F}$ \\
\hline $\begin{array}{l}\text { Region comprising left inferior longitudinal } \\
\text { and fronto-occipital fasciculi and forceps } \\
\text { major of the CC }\end{array}$ & $-24,-76,-2$ & -2.24 & 0.000006 & 552 & $12 / 12$ & Yes & - \\
\hline
\end{tabular}

Abbreviations: CC, corpus callosum; FA, fractional anisotropy; SLF, superior longitudinal fasciculus; WMV, white matter volume.

a Robustness based on jack-knife analysis, replication after discarding pediatric/adolescent samples, and funnel plots from the WMV and FA meta-analyses.

${ }^{\text {b}}$ The abnormality did not reach statistical significance in combination/s without the study or studies within brackets.

${ }^{\mathrm{c}}$ Corresponding multimodal finding.

fronto-occipital fasciculus. Findings were particularly robust in anterior midline WM structures (mainly the crossing between the anterior part parts of the cingulum bundle and the body of CC), which showed both increased WMV and decreased FA. These results remained unaltered after the exclusion of pediatric samples. Thus, our results primarily relate to adult OCD patients. Owing to insufficient number of available studies, we were unable to draw specific conclusions regarding WM alterations in pediatric OCD.

The decrease of FA in midline WM structures may suggest an increase of fiber crossing in OCD, and the increase of WMV may suggest that this is because of an increase of volume of the non-dominant tract. As exemplified in Figure 2, WMV and FA can both be increased or decreased in areas where fiber tracts are organized in parallel. However, in the case of fiber crossing, WMV can increase, whereas FA decreases. Among the two tracts crossing the midline WM areas, one is thicker (ie, dominant) and the other(s) thinner (ie, non-dominant) in healthy controls. The predominance of the dominant tract forces the water to mainly flow in only one direction (ie, that of the dominant tract), translating in a relatively high FA. If this interpretation were correct, patients with OCD would have an increase of the thickness of a non-dominant tract, resulting in increased volume, as well as in water flowing in two directions rather than one (ie, those of the two tracts) and thus lower FA. However, other interpretations might be also considered, as changes in WMV and FA could also be due to, for example, changes in membrane permeability or in the presence of non-axonal components such as other cells, vessels or interstitial fluid.

The main WM abnormalities reported here are spatially very close to the gray matter regions that have been consistently found to be abnormal (ie, reduced volumes in cingulate/dorsomedial prefrontal cortices) in previous meta-analyses (Radua and Mataix-Cols, 2009; Radua et al, 2010) and a recent mega-analysis (de Wit et al, 2013) of
OCD studies. These cortical regions have also been reported to be abnormally activated in functional magnetic resonance studies of patients with OCD, including resting state studies (Swedo et al, 1989), symptom provocation studies (Rauch et al, 1994), and studies using tasks requiring inhibitory control (van den Heuvel et al, 2005). These regions are also anatomically very close to one of the major neurosurgical treatments for OCD, cingulotomy (Ballantine et al, 1987). Thus, the findings of this meta-analysis add further evidence to the concept of a structural and functional abnormality in the anterior cingulate bundle and adjacent cortex in OCD. Rather than being specific to OCD, it is tenable that these abnormalities may be shared with other anxiety and mood disorders (Radua et al, 2010; van Tol et al, 2010).

Interestingly, meta-regression analysis showed that the decrease of FA in these midline structures was most prominent in samples that had higher percentages of medicated patients. Although the effects of selective reuptake inhibitors on WM parameters are not well understood, these findings may be consistent with previous work indicating that drug-treated OCD patients have widespread increase of ( $\lambda_{2}$ and $\lambda_{3}$-averaged) radial diffusivity in adjacent CC/ cingulate gyrus, compared with drug-naive patients and healthy controls (Benedetti et al, 2013). Reduced FA with increased mean and radial diffusivity suggests changes in myelination; this would be consistent with drug treatments modifying the structure of cell membranes and myelin sheaths by various mechanisms including oligodendrocyte differentiation and remyelination. However, other interpretations may also be considered, for example, medication could induce an increase of crossing fibers, and potential selection bias should also be taken into account. On the other hand, there is some evidence that WM alterations in OCD may be partially reversible with pharmacotherapy (Fan et al, 2012; Yoo et al, 2007), although these small studies lacked a control (placebo) group and therefore these 


\section{MAIN FINDING}

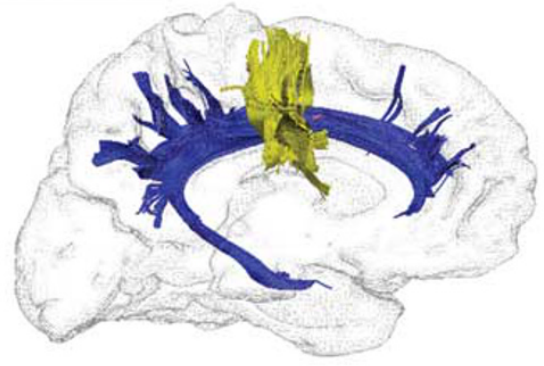

Increase of white matter volume (WMV) but decrease of fractional anisotropy (FA)

(B) Crossing between the anterior body of the corpus callosum (yellow) and cingulum bundle (dark blue)

Peak at $-8,-4,-38$. Slice: $y=-4$

\section{META-REGRESSION}
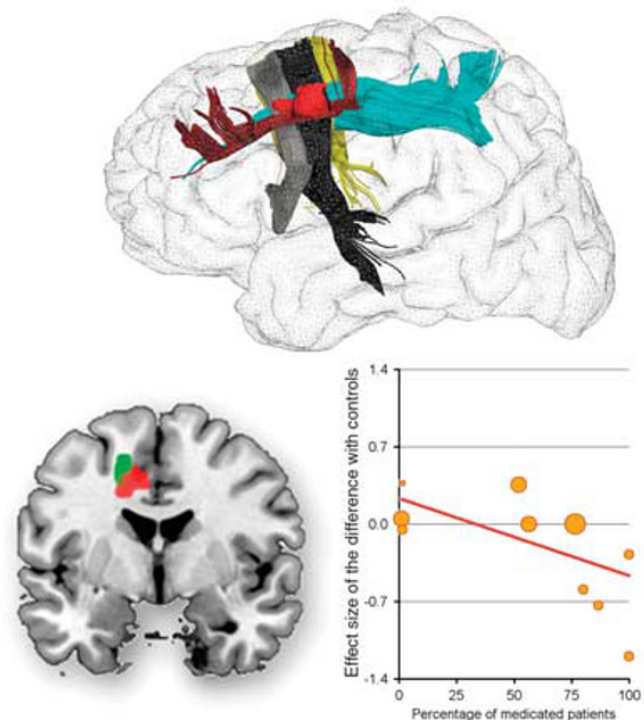

\section{OTHER FINDINGS}

Increase of both, white matter volume (WMV) and fractional anisotropy (FA)
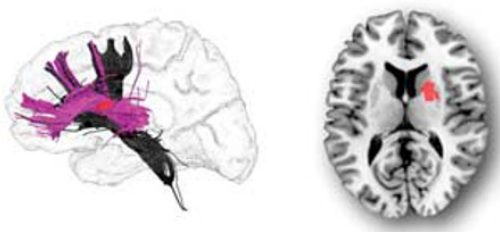

(A) Parallel right anterior thalamic radiation (pink) and cortico spinal tract (black) through the internal capsule

Peak at $20,4,14$. Slice: $z=14$

Increase of white matter volume (WMV) but decrease of fractional anisotropy (FA)

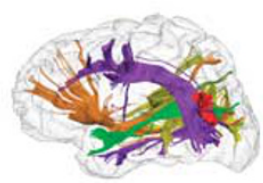

(C) Crossing between the middle longitudinal fasciculus (green), inferior fronto-occipital fasciculus (orange), forceps major of the corpus callosum (yellow) and arcuate fasciculus (purple)

Peak at $-30,-50,16$. Slice: $z=16$

(D) Crossing between the anterior body of the corpus callosum (yellow), cingulum bundle (dark blue) and part of the superior longitudinal fasciculus (light blue)

Peak at $10,24,26$. Slice: $x=10$

Decrease of white matter volume (WMV) but increase of fractional anisotropy (FA)
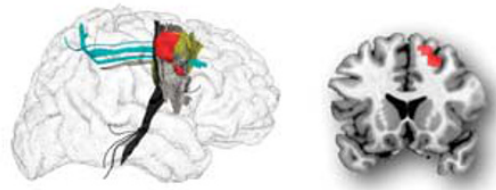

(E) Crossing between the superior longitudinal fasciculus (light blue), cortico spinal tract (black), frontal aslant tract (grey) and anterior body of the corpus callosum (yellow)

Peak at $12,16,48$. Slice: $\mathrm{y}=16$

Decrease of $b o t h$, white matter volume (WMV) and fractional anisotropy (FA)

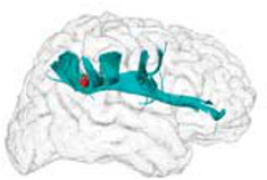

(F) Right superior longitudinal fasciculus (light blue)

Peak at $32,-44,36$. Slice: $y=-44$

Figure I Regions with abnormal white matter volume (WMV) and fractional anisotropy (FA) in patients with obsessive-compulsive disorder (OCD), and effects of medication use on the observed FA abnormalities. Regions with abnormal WMV and FA are displayed in red, and meta-regression results (slice $y=-2$ ) in green. Superior longitudinal fasciculus (light blue); cingulum bundle (dark blue); corpus callosum (yellow); anterior thalamic radiation (pink); corticospinal tract (black); frontal aslant tract (gray); frontal superior longitudinal (dark red); middle longitudinal fasciculus (green); inferior fronto-occipital fasciculus (orange); arcuate fasciculus (purple). Effect sizes used to create the meta-regression plot were extracted from the peak of maximum slope significance, and may thus overestimate the effect that would be observed in a broader region.

changes could not be unequivocally linked to the pharmacological treatment itself. A longitudinal study in patients with multiple sclerosis also found that FA may be modulated by fluoxetine (Sijens et al, 2008), although changes did not reach statistical significance. The impact of medication on WM remains an area of research for the future.

As the SLF connects the middle frontal gyrus/dorsolateral prefrontal cortex with the superior parietal lobule, the results of our meta-analysis also support a growing body of evidence that other regions outside the traditional cortico- striatal loops, such as parietal cortex, may be involved in OCD (Menzies et al, 2008a; Radua and Mataix-Cols, 2009; Radua et al, 2010).

\section{Strengths and Limitations}

Strengths of this study are the use of novel techniques that combine the positive features from coordinate metaanalytic approaches with those from standard meta-analytic methods, the use of a multimodal approach, which provides 
PARALLEL FIBER TRACT

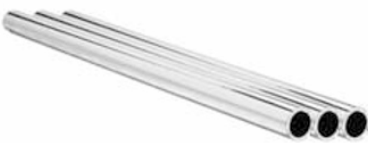

An increase of the number or diameter of axons results in an increase of both volume (more amount of white matter) and fractional anisotropy (water more restricted to flow in only one direction).

Increase of the tract: $\uparrow \mathrm{WMV}, \uparrow \mathrm{FA}$

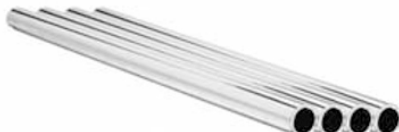

Increase of fiber crossing: $\uparrow \mathrm{WMV}, \downarrow \mathrm{FA}$

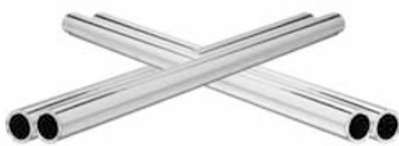

An increase of the number or diameter of axons of the non-dominant trac results in an increase of volume (more amount of white matter) and a decrease of fractional anisotropy (water restricted to flow in two directions.

decrease of fractional anisotropy
rather than mainly in only one)
Decrease of the tract: $\downarrow$ WMV $\downarrow$ FA

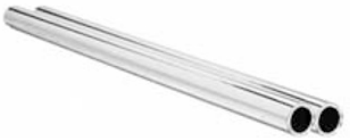

A decrease of the number or diameter of axons results in a decrease of both volume (less amount of white matter) and fractional anisotropy (water more free to flow in other directions).
FIBER CROSSING

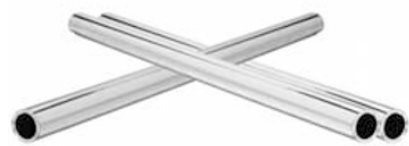

Figure 2 White matter volume (WMV) and fractional anisotropy (FA) in a parallel fiber tract and in a simple fiber crossing, where the non-dominant tract (ie, the tract with lower volume) is drawn darker. Please note that Figure 2 represents a simple scenario in which there is an increase or decrease of the number or diameter of axons of only one of the tracts. More complex scenarios might be also considered, involving abnormalities in more than two tracts or due to, for example, changes in membrane permeability or in the presence of non-axonal components such as other cells, vessels or interstitial fluid.

a unique multimodal view of WM alterations in OCD, and the inclusion of previously unpublished data. Of relevance, we were able to obtain the SPMs from two of the studies included, thus substantially optimizing the statistical power of the meta-analysis. For example, in the ES-SDM validation study, sensitivity increased from 55 to $73 \%$ with inclusion of just one SPM and to $87 \%$ with inclusion of two SPMs (Radua et al, 2012b). In addition, the findings were projected into a novel high-resolution diffusion-weighted imaging data set in order to facilitate a more precise, yet approximate identification of the WM tracts involved (Thiebaut de Schotten et al, 2012).

The study also had several limitations. First, the total sample of subjects was relatively small and we could not include TBSS studies (comprising 127 patients and 129 controls). Second, there were too few pediatric OCD studies for separate meta-analyses. This is particularly important, as the trajectories of brain maturation in OCD are currently unknown and it is tenable that the WM tracts implicated in pediatric $v s$ adult OCD cases may differ. We were, however, able to conclude that our main findings remain broadly unchanged after limiting our analyses to adult samples only. Third, we were only able to include a few clinical variables in the meta-regression models as these were inconsistently reported in the original studies. Furthermore, as mentioned earlier, the results of the meta-regression analyses should be interpreted cautiously because they were either driven by only few studies or did not clearly overlap with the betweengroup differences found in the main analyses. Fourth, we were able to approximately localize regions of potential abnormality, but not to distinguish which particular tract or tracts of those crossing these regions were abnormal. The relatively more precise localization obtained with TBSS studies could potentially help isolate specific tracts; a metaanalysis of TBSS studies is recommended once sufficient numbers of high quality studies become available. Fifth, results defined as 'less robust' (see Table 2) should be taken with caution. Sixth, there was some potential methodological heterogeneity as some WMV studies included a modulation step, whereas others did not. However, it must be noted that modulated and unmodulated volumetric images are essentially similar, with the only difference that the former may be more statistically powerful for detecting macroscopic differences, whereas the latter may be more suitable for the detection of smaller, mesoscopic abnormalities; see Radua et al (2013a) for a review and empirical simulations. Similarly, some studies used corrected $P$ values, whereas others did not. This might affect the precision of the estimation of the effect sizes, with the maps being better recreated when a large number of reported peaks is available-independently of whether they are statistically significant or not. Finally, there is growing evidence that OCD is a heterogeneous disorder (MataixCols et al, 2005), with specific neurocircuitry partially underpinning different symptom dimensions (Mataix-Cols and van den Heuvel, 2006). For instance, the contamination/washing dimension has been reported to be associated with parietal WM abnormalities, whereas harm/checking and symmetry/ordering with temporal WM abnormalities (van den Heuvel et al, 2009). Additional work with larger and better-characterized samples is needed in order to meta-analyze the WM correlates of such symptom dimensions.

\section{Conclusions}

Keeping the above caveats in mind, our results suggest that patients with OCD may have widespread WM abnormalities, which are particularly prominent in anterior midline structures. The findings are broadly consistent with both the classic fronto-striatal model of the disorder and also 
with more recent 'systems' approaches, which emphasize the implication of multiple other brain systems (frontolimbic, fronto-parietal) in OCD. The potential effects of medication on WM in OCD should be investigated further. Longitudinal studies of brain maturation from childhood to adulthood are also needed.

\section{FUNDING AND DISCLOSURE}

Dr Canales-Rodríguez, Dr Catani and Dr Mataix-Cols declare that, except for income received from their primary employers, no financial support or compensation has been received from any individual or corporate entity over the past 3 years for research or professional service and there are no personal financial holdings that could be perceived as constituting a potential conflict of interest. Dr Radua has been a clinical rater in Roche clinical trials. Dr Grau has received funds from Alicia Koplowitz Foundation. Dr van den Heuvel has received a speaker honorarium from Eli Lilly. Dr Thiebaut de Schotten has received a research grant from Shire. Dr Stein has received research grants and/or consultancy honoraria from Biocodex, Eli-Lilly, GlaxoSmithKline, Lundbeck, and Servier.

\section{ACKNOWLEDGEMENTS}

We thank all the authors of the included studies and very specially Drs Gong, Kwon, Lazaro, Murat, Nakao, Nakamae, Perani, Pujol, Rauch, Szeszko and colleagues for kindly sharing unpublished peak data and SPMs for inclusion in this meta-analysis. We also thank Dr Nemat Jaafari who helped retrieving papers in the early phases of this study. This work was supported by Instituto de Salud Carlos III (Río Hortega research contract to Dr Radua, CM11/00024). The funding organization had no role in the study design, data collection and analysis, or manuscript approval.

\section{REFERENCES}

Admon R, Bleich-Cohen M, Weizmant R, Poyurovsky M, Faragian S, Hendler T (2012). Functional and structural neural indices of risk aversion in obsessive-compulsive disorder (OCD). Psychiatry Res 203: 207-213.

Avants BB, Epstein CL, Grossman M, Gee JC (2008). Symmetric diffeomorphic image registration with cross-correlation: evaluating automated labeling of elderly and neurodegenerative brain. Med Image Anal 12: 26-41.

Ballantine HT Jr, Bouckoms AJ, Thomas EK, Giriunas IE (1987). Treatment of psychiatric illness by stereotactic cingulotomy. Biol Psychiatry 22: 807-819.

Benedetti F, Giacosa C, Radaelli D, Poletti S, Pozzi E, Dallaspezia S et al (2013). Widespread changes of white matter microstructure in obsessive-compulsive disorder: effect of drug status. Eur Neuropsychopharmacol 23: 581-593.

Cannistraro PA, Makris N, Howard JD, Wedig MM, Hodge SM, Wilhelm S et al (2007). A diffusion tensor imaging study of white matter in obsessive-compulsive disorder. Depress Anxiety 24: 440-446.

Carmona S, Bassas N, Rovira M, Gispert JD, Soliva JC, Prado M et al (2007). Pediatric OCD structural brain deficits in conflict monitoring circuits: a voxel-based morphometry study. Neurosci Lett 421: 218-223.
Catani M, Dell'acqua F, Vergani F, Malik F, Hodge H, Roy P et al (2012). Short frontal lobe connections of the human brain. Cortex 48: 273-291.

Daniele A, Bartolomeo P, Cassetta E, Bentivoglio AR, Gainotti G, Albanese A et al (1997). Obsessive-compulsive behaviour and cognitive impairment in a parkinsonian patient after left putaminal lesion. J Neurol Neurosurg Psychiatry 62: 288-289.

de Wit SJ, Alonso P, Schweren L, Mataix-Cols D, Lochner C, Menchon JM et al (2013). Multicenter voxel-based morphometry mega-analysis of structural brain scans in obsessive-compulsive disorder. Am J Psychiatry doi:10.1176/appi.ajp.2013.13040574.

Duran FL, Hoexter MQ, Valente AA Jr, Miguel EC, Busatto GF (2009). Association between symptom severity and internal capsule volume in obsessive-compulsive disorder. Neurosci Lett 452: 68-71.

Fan Q, Yan X, Wang J, Chen Y, Wang X, Li C et al (2012). Abnormalities of white matter microstructure in unmedicated obsessive-compulsive disorder and changes after medication. PLoS One 7: e35889.

Fontenelle LF, Harrison BJ, Yucel M, Pujol J, Fujiwara H, Pantelis C (2009). Is there evidence of brain white-matter abnormalities in obsessive-compulsive disorder?: a narrative review. Top Magn Reson Imaging 20: 291-298.

Garibotto V, Scifo P, Gorini A, Alonso CR, Brambati S, Bellodi L et al (2010). Disorganization of anatomical connectivity in obsessive compulsive disorder: a multi-parameter diffusion tensor imaging study in a subpopulation of patients. Neurobiol Dis 37: 468-476.

Gruner P, Vo A, Ikuta T, Mahon K, Peters BD, Malhotra AK et al (2012). White matter abnormalities in pediatric obsessivecompulsive disorder. Neuropsychopharmacology 37: 2730-2739.

Ha TH, Kang DH, Park JS, Jang JH, Jung WH, Choi JS et al (2009). White matter alterations in male patients with obsessivecompulsive disorder. Neuroreport 20: 735-739.

Harrison BJ, Soriano-Mas C, Pujol J, Ortiz H, Lopez-Sola M, Hernandez-Ribas R et al (2009). Altered corticostriatal functional connectivity in obsessive-compulsive disorder. Arch Gen Psychiatry 66: 1189-1200.

Koprivova J, Hor cek J, Tintera J, Prasko J, Raszka M, Ibrahim I et al (2009). Medial frontal and dorsal cortical morphometric abnormalities are related to obsessive-compulsive disorder. Neurosci Lett 464: 62-66.

Lazaro L, Castro-Fornieles J, Cullell C, Andres S, Falcon C, Calvo R et al (2011). A voxel-based morphometric MRI study of stabilized obsessive-compulsive adolescent patients. Prog Neuropsychopharmacol Biol Psychiatry 35: 1863-1869.

Li F, Huang X, Yang Y, Li B, Wu Q, Zhang T et al (2011). Microstructural brain abnormalities in patients with obsessivecompulsive disorder: diffusion-tensor MR imaging study at 3.0T. Radiology 260: 216-223.

Mataix-Cols D, Rosario-Campos MC, Leckman JF (2005). A multidimensional model of obsessive-compulsive disorder. $\mathrm{Am} \mathrm{J}$ Psychiatry 162: 228-238.

Mataix-Cols D, van den Heuvel OA (2006). Common and distinct neural correlates of obsessive-compulsive and related disorders. Psychiatr Clin North Am 29: 391-410, viii.

Matsumoto R, Ito H, Takahashi H, Ando T, Fujimura Y, Nakayama K et al (2010). Reduced gray matter volume of dorsal cingulate cortex in patients with obsessive-compulsive disorder: a voxel-based morphometric study. Psychiatry Clin Neurosci 64: 541-547.

Menzies L, Chamberlain SR, Laird AR, Thelen SM, Sahakian BJ, Bullmore ET (2008a). Integrating evidence from neuroimaging and neuropsychological studies of obsessive-compulsive disorder: the orbitofronto-striatal model revisited. Neurosci Biobehav Rev 32: 525-549.

Menzies L, Williams GB, Chamberlain SR, Ooi C, Fineberg N, Suckling J et al (2008b). White matter abnormalities in patients with obsessive-compulsive disorder and their first-degree relatives. Am J Psychiatry 165: 1308-1315. 
Milad MR, Rauch SL (2011). Obsessive-compulsive disorder: beyond segregated cortico-striatal pathways. Trends Cogn Sci 16: $43-51$.

Nakamae T, Narumoto J, Shibata K, Matsumoto R, Kitabayashi Y, Yoshida $\mathrm{T}$ et al (2008). Alteration of fractional anisotropy and apparent diffusion coefficient in obsessive-compulsive disorder: a diffusion tensor imaging study. Prog Neuropsychopharmacol Biol Psychiatry 32: 1221-1226.

Peng Z, Lui SS, Cheung EF, Jin Z, Miao G, Jing J et al (2012). Brain structural abnormalities in obsessive-compulsive disorder: converging evidence from white matter and grey matter. Asian $J$ Psychiatr 5: 290-296.

Peters BD, Szeszko PR, Radua J, Ikuta T, Gruner P, DeRosse P et al (2012). White matter development in adolescence: diffusion tensor imaging and meta-analytic results. Schizophr Bull 38: $1308-1317$.

Pujol J, Soriano-Mas C, Alonso P, Cardoner N, Menchon JM, Deus J et al (2004). Mapping structural brain alterations in obsessivecompulsive disorder. Arch Gen Psychiatry 61: 720-730.

Radua J, Borgwardt S, Crescini A, Mataix-Cols D, MeyerLindenberg A, McGuire PK et al (2012a). Multimodal metaanalysis of structural and functional brain changes in first episode psychosis and the effects of antipsychotic medication. Neurosci Biobehav Rev 36: 2325-2333.

Radua J, Canales-Rodríguez EJ, Pomarol-Clotet E, Salvador R (2013a). Validity of modulation and optimal settings for advanced voxel-based morphometry. Neuroimage 86: 81-90.

Radua J, Mataix-Cols D (2009). Voxel-wise meta-analysis of grey matter changes in obsessive-compulsive disorder. Br J Psychiatry 195: 393-402.

Radua J, Mataix-Cols D, Phillips ML, El-Hage W, Kronhaus DM, Cardoner $\mathrm{N}$ et al (2012b). A new meta-analytic method for neuroimaging studies that combines reported peak coordinates and statistical parametric maps. Eur Psychiatry 27: 605-611.

Radua J, Romeo M, Mataix-Cols D, Fusar-Poli P (2013b). A general approach for combining voxel-based meta-analyses conducted in different neuroimaging modalities. Curr Med Chem 20: 462-466.

Radua J, van den Heuvel OA, Surguladze S, Mataix-Cols D (2010). Meta-analytical comparison of voxel-based morphometry studies in obsessive-compulsive disorder $v s$ other anxiety disorders. Arch Gen Psychiatry 67: 701-711.

Radua J, Via E, Catani M, Mataix-Cols D (2011). Voxel-based metaanalysis of regional white-matter volume differences in autism spectrum disorder versus healthy controls. Psychol Med 41: 1539-1550.

Rauch SL, Jenike MA, Alpert NM, Baer L, Breiter HC, Savage CR et al (1994). Regional cerebral blood flow measured during symptom provocation in obsessive-compulsive disorder using oxygen 15-labeled carbon dioxide and positron emission tomography. Arch Gen Psychiatry 51: 62-70.
Riffkin J, Yucel M, Maruff P, Wood SJ, Soulsby B, Olver J et al (2005). A manual and automated MRI study of anterior cingulate and orbito-frontal cortices, and caudate nucleus in obsessivecompulsive disorder: comparison with healthy controls and patients with schizophrenia. Psychiatry Res 138: 99-113.

Rotge JY, Langbour N, Guehl D, Bioulac B, Jaafari N, Allard M et al (2009). Gray matter alterations in obsessive-compulsive disorder: an anatomic likelihood estimation meta-analysis. Neuropsychopharmacology 35: 686-691.

Saxena S, Rauch SL (2000). Functional neuroimaging and the neuroanatomy of obsessive-compulsive disorder. Psychiatr Clin North Am 23: 563-586.

Sijens PE, Mostert JP, Irwan R, Potze JH, Oudkerk M, De Keyser J (2008). Impact of fluoxetine on the human brain in multiple sclerosis as quantified by proton magnetic resonance spectroscopy and diffusion tensor imaging. Psychiatry Res 164: 274-282.

Swedo SE, Schapiro MB, Grady CL, Cheslow DL, Leonard HL, Kumar A et al (1989). Cerebral glucose metabolism in childhood-onset obsessive-compulsive disorder. Arch Gen Psychiatry 46: 518-523.

Szeszko PR, Ardekani BA, Ashtari M, Malhotra AK, Robinson DG, Bilder RM et al (2005). White matter abnormalities in obsessivecompulsive disorder: a diffusion tensor imaging study. Arch Gen Psychiatry 62: 782-790.

Thiebaut de Schotten M, Dell'Acqua F, Valabregue R, Catani M (2012). Monkey to human comparative anatomy of the frontal lobe association tracts. Cortex 48: 82-96.

Togao O, Yoshiura T, Nakao T, Nabeyama M, Sanematsu H, Nakagawa A et al (2010). Regional gray and white matter volume abnormalities in obsessive-compulsive disorder: a voxel-based morphometry study. Psychiatry Res 184: 29-37.

van den Heuvel OA, Remijnse PL, Mataix-Cols D, Vrenken H, Groenewegen HJ, Uylings HB et al (2009). The major symptom dimensions of obsessive-compulsive disorder are mediated by partially distinct neural systems. Brain 132(Pt 4): 853-868.

van den Heuvel OA, Veltman DJ, Groenewegen HJ, Cath DC, van Balkom AJ, van Hartskamp J et al (2005). Frontal-striatal dysfunction during planning in obsessive-compulsive disorder. Arch Gen Psychiatry 62: 301-309.

van Tol MJ, van der Wee NJ, van den Heuvel OA, Nielen MM, Demenescu LR, Aleman A et al (2010). Regional brain volume in depression and anxiety disorders. Arch Gen Psychiatry 67: 10021011.

Yoo SY, Jang JH, Shin YW, Kim DJ, Park HJ, Moon WJ et al (2007). White matter abnormalities in drug-naive patients with obsessive-compulsive disorder: a diffusion tensor study before and after citalopram treatment. Acta Psychiatr Scand 116: 211-219.

Yoo SY, Roh MS, Choi JS, Kang DH, Ha TH, Lee JM et al (2008). Voxel-based morphometry study of gray matter abnormalities in obsessive-compulsive disorder. J Korean Med Sci 23: 24-30.

Supplementary Information accompanies the paper on the Neuropsychopharmacology website (http://www.nature.com/npp) 